\title{
Effectiveness of an influenza vaccine programme for care home staff to prevent death, morbidity, and health service use among residents: cluster randomised controlled trial
}

Andrew C Hayward, Richard Harling, Sally Wetten, Anne M Johnson, Susan Munro, Julia Smedley, Shahed Murad, John M Watson

\begin{abstract}
Objective To determine whether vaccination of care home staff against influenza indirectly protects residents.

Design Pair matched cluster randomised controlled trial.

Setting Large private chain of UK care homes during the winters of 2003-4 and 2004-5.

Participants Nursing home staff $(\mathrm{n}=1703)$ and residents $(\mathrm{n}=2604)$ in 44 care homes (22 intervention homes and 22 matched control homes).

Interventions Vaccination offered to staff in intervention homes but not in control homes.

Main outcome measures The primary outcome was all cause mortality of residents. Secondary outcomes were influenza-like illness and health service use in residents.

Results In 2003-4 vaccine coverage in full time staff was 48.2\% $(407 / 884)$ in intervention homes and 5.9\% (51/859) in control homes. In 2004-5 uptake rates were 43.2\% (365/844) and 3.5\% $(28 / 800)$. National influenza rates were substantially below average in 2004-5. In the 2003-4 period of influenza activity significant decreases were found in mortality of residents in intervention homes compared with control homes (rate difference -5.0 per 100 residents, $95 \%$ confidence interval -7.0 to -2.0$)$ and in influenza-like illness $(\mathrm{P}=0.004)$, consultations with general practitioners for influenza-like illness $(\mathrm{P}=0.008)$, and admissions to hospital with influenza-like illness $(\mathrm{P}=0.009)$. No significant differences were found in 2004-5 or during periods of no influenza activity in 2003-4. Conclusions Vaccinating care home staff against influenza can prevent deaths, health service use, and influenza-like illness in residents during periods of moderate influenza activity.

Trial registration National Research Register N0530147256.
\end{abstract}

\section{Introduction}

Influenza is an important cause of mortality, morbidity, and health service use in elderly patients. ${ }^{1}$ The virus can spread particularly rapidly in care homes, ${ }^{2}$ with attack rates ranging from $20-40 \%{ }^{3-7}$ but potentially reaching $60 \%$ of residents. ${ }^{8}$ Complications are common, with admission rates to hospital often exceeding $10 \% \%^{4-6} 8$ and case fatality rates often exceeding $5 \% \%^{4-8}$ and reaching $55 \%{ }^{3}$ Vaccination of care home residents against influenza is effective in preventing respiratory illness, admissions to hospital, and death. ${ }^{9-11}$ The immune response to influenza vaccine in elderly patients (especially those with comorbidities) is, however, reduced, so that protection is only $50-70 \% .{ }^{12}{ }^{13}$ Resi- dents are therefore vulnerable to influenza outbreaks even when vaccination coverage is high. ${ }^{3-5} 7$

In many countries it is recommended that acute hospitals offer influenza vaccine to healthcare workers annually. ${ }^{14}$ Employers in the United Kingdom are advised to consider providing vaccination for care home staff, but most do not. ${ }^{15}$ Evidence shows that vaccination of healthcare workers can reduce serologically confirmed influenza by nearly $90 \%$ in those vaccinated.$^{16}$ An indirect effect may also exist whereby immune staff do not infect patients. ${ }^{17}{ }^{18}$ Two previous cluster randomised controlled trials showed that influenza vaccination of healthcare workers on wards for the care of elderly people in Scotland led to a decrease in mortality among patients. ${ }^{17}{ }^{18}$ Results have been questioned owing to the relatively small number of wards randomised (which led to unbalanced randomisation) and because it was not possible to show that the reductions in mortality were related temporally to influenza activity on the wards or in the community. ${ }^{19}$ We studied the effect of vaccinating care home staff against influenza on mortality, health service use, and influenza-like illness among residents. To overcome some of the methodological limitations of previous studies we randomised a large number of units and balanced these on baseline characteristics. We used cluster randomisation to look for indirect effects of vaccination and because the intervention was best applied at the level of the care home. ${ }^{20}$ We compared the effectiveness of the intervention during periods with differing levels of influenza activity in the community as this is likely to influence the effect size. The study is reported according to the guidelines of the consolidated standards of reporting trials for cluster randomised controlled trials. ${ }^{21}$

\section{Methods}

We carried out a pair matched cluster randomised controlled trial of promotion and delivery of influenza vaccine to care home staff over the winters of 2003-4 and 2004-5, with collection of aggregate data on outcomes among residents. The study was carried out in a large private chain of UK care homes. Residents were predominantly elderly and required a mixture of nursing and residential care. The company's policy (in common with most UK care homes) was not to offer staff influenza vaccination. The homes routinely offer influenza vaccine to all residents and

Forest plots for rate differences during year 1 are on bmj.com 
arrange this through local general practitioners. We excluded homes in Scotland and Wales for logistical reasons.

\section{Intervention}

For the purposes of the study the company agreed to adopt a policy for influenza vaccination of staff in randomly selected intervention homes while maintaining their usual policy of not actively promoting staff vaccination in control homes. Lead nurses in each of the intervention homes were trained to promote influenza vaccine to staff. They were encouraged to act as advocates for vaccination and to use word of mouth, leaflets, and posters to promote vaccination. Staff in intervention homes were eligible for vaccination and were sent a letter explaining the study and the potential benefits of influenza vaccination. The lead nurse liaised with a local occupational health service to arrange for three vaccination sessions within the homes in October, including at least one session during a night shift to maximise uptake. Staff in control homes were sent a letter informing them of the study and advising them of the Department of Health recommendation that adults with chronic illness should be vaccinated by their general practitioner. We did not seek to influence vaccination of residents.

We hypothesised that the vaccine promotion programme for staff would reduce transmission of influenza to residents and therefore reduce influenza-like illness and associated deaths and health service use in residents during and immediately after periods of influenza virus activity but not at other times.

\section{Outcomes}

Outcomes were measured in residents and collected as aggregate data within each home. The primary outcome was all cause mortality of residents. Secondary outcomes were influenza-like illness, mortality with influenza-like illness, admissions to hospital from any cause, admissions to hospital with influenza-like illness, and consultations with a general practitioner for influenza-like illness. The lead nurses at each home were trained to collect daily data about the numbers of residents at the home and the numbers who experienced primary or secondary outcomes. Influenza-like illness was defined as a fever of $37.8^{\circ} \mathrm{C}$ or more (measured orally), or an acute deterioration in physical or mental ability, plus either new onset of one or more respiratory symptoms or an acute worsening of a chronic condition involving respiratory symptoms. This case definition was adapted from others used in this setting. ${ }^{4-7}$ Since elderly people often do not have a fever, our definition did not require a raised temperature. Data were returned weekly. Data collection took place from 3 November 2003 to 28 March 2004 and from 1 November 2004 to 27 March 2005. Only aggregate nonidentifiable data were collected.

\section{Sample size and randomisation}

Sample size calculations for cluster randomised controlled trials ${ }^{22}$ were applied to data from a pilot study and previous studies. ${ }^{17} 18$ To detect a reduction in all cause mortality from $15 \%$ to $10 \%$ (intracluster variance $2.3 \%$ ) with $90 \%$ power at the $5 \%$ significance level we determined that we required 20 pairs of homes, with an average of 50 residents each, studied for one winter. The study was carried out over two consecutive years to minimise the possibility of negative results because of low influenza activity.

For all homes we obtained data on the number of residents, the proportion requiring high dependency care, and mortality. We placed homes into matched pairs within three regions (northern, central, and southern England) on the basis of the following order of priority: size of home, percentage of high

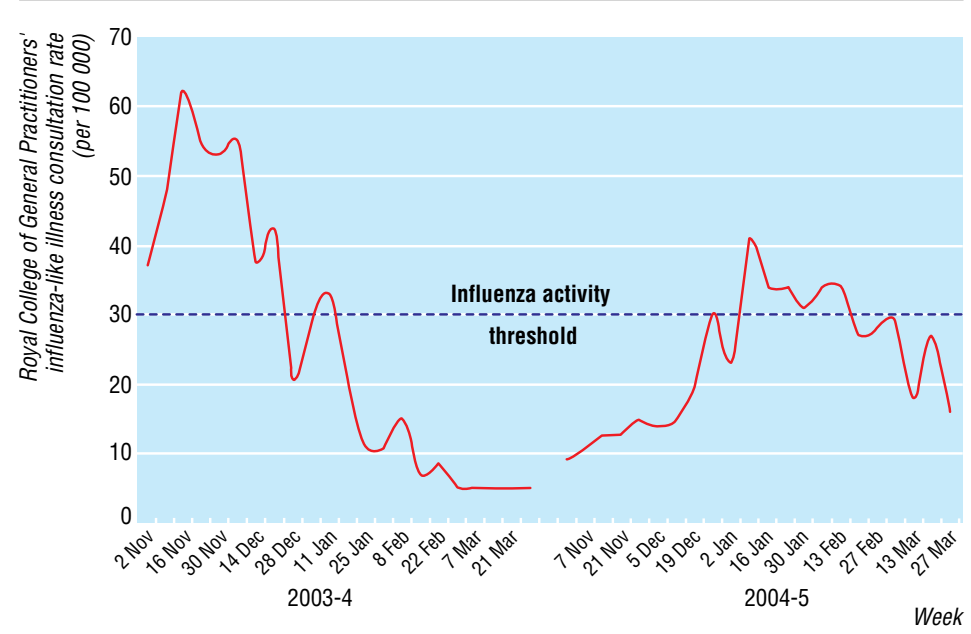

Fig 1 Royal College of General Practitioners' consultation rate for influenza-like illness (consultations per 100000 population) and periods of influenza activity

dependency, and mortality of residents. We then selected the 25 most closely matching pairs of homes. A researcher blinded to the home's identity and characteristics carried out randomisation within these pairs using random number tables.

\section{Statistical analysis}

We used national data from the Royal College of General Practitioners' sentinel surveillance scheme to divide the study into periods of influenza activity and no activity. ${ }^{23}$ The start of the period of influenza activity was defined as the beginning of the week in which the weekly consultation rate rose above 30 per 100000 (standard cut-off point for defining normal seasonal influenza activity). The end was defined as one week after the weekly consultation rate returned and remained below this baseline level for influenza-like illness and for general practitioner consultations for influenza-like illness, and two weeks after the consultation rate fell below baseline for admissions to hospital and deaths, thus taking into account a plausible timescale for development and progression of disease (fig 1).

We analysed outcomes at a cluster level rather than individual level using aggregate data for each cluster. To take account of the matched clustered design we used a random effects meta-analysis. ${ }^{24}$ This treated the results from each pair of homes as a separate study and provided a pooled estimate of effect weighted for the size of homes and the size of the effects and their standard errors. We calculated the outcome rates per resident period for each home during periods of influenza activity and no activity. The rates were measures based on person time where the denominator was the average number of residents during the period of interest (calculated as the number of occupied bed days during the period divided by the number of days in the period) and the numerator was the number of events in these residents during the period. For each pair of homes we calculated the rate difference (intervention minus control); a negative rate difference indicating that the intervention prevented events. We used RevMan software to produce forest plots of rate differences for each pair of homes and weighted estimates of the overall rate difference, 95\% confidence intervals, and levels of statistical significance. To test for interaction between year and intervention we used a multilevel Poisson model.

When significant protection of residents was observed we calculated the number of staff vaccinations needed to prevent one event in residents (number needed to treat) as number of 
Research

Table 1 Baseline characteristics of residents in intervention and control homes

\begin{tabular}{|c|c|c|c|c|}
\hline \multirow{2}{*}{ Characteristic } & \multicolumn{2}{|c|}{ Intervention homes } & \multicolumn{2}{|c|}{ Control homes } \\
\hline & 2003-4 & $2004-5$ & 2003-4 & $2004-5$ \\
\hline No of residents & 1233 & 1270 & 1371 & 1391 \\
\hline Mean age (years) & 83.0 & 82.7 & 82.6 & 83.0 \\
\hline No (\%) of women & $866(70.2)$ & $897(70.6)$ & $972(70.9)$ & $976(70.2)$ \\
\hline No (\%) highly dependent* & $444(36.0)$ & $627(49.4)$ & $568(41.4)$ & $640(46.0)$ \\
\hline No (\%) vaccinated & 964 (78.2) & 895 (70.5) & $979(71.4)$ & 989 (71.1) \\
\hline
\end{tabular}

${ }^{*}$ Classified according to nursing home chain's in-house scoring system. Patients classed as highly dependent (for example, bedridden or severely demented patients) required more intensive care.

vaccinations given in all intervention homes divided by the average number of residents in all intervention homes multiplied by the weighted rate difference.

\section{Results}

Figure 1 shows the Royal College of General Practitioners' consultation rate for 2003-4 and 2004-5 and our periods of influenza activity for influenza-like illness and general practitioner consultations for influenza-like illness, admissions to hospital, and deaths. According to surveillance, influenza activity was below average in $2003-4$ but nearly double that in $2004-5 .{ }^{23}$ In both years laboratory surveillance confirmed that influenza A H3N2 was circulating during these periods (Fujian subtype in 2003-4 and Wellington subtype in 2004-5).
One care home withdrew after randomisation, and two were unable to provide regular data on outcomes. These homes and their matching homes were excluded, leaving 22 pairs (fig 2). Analyses relate to these 22 pairs (no outcome data were available for the excluded homes so an intention to treat analysis was not possible). No significant differences were found between baseline characteristics of the excluded pairs and the remaining homes. Influenza vaccine coverage among full time staff in intervention homes was $48.2 \%$ in 2003-4 and 43.2\% in 2004-5 compared with $5.9 \%$ and $3.5 \%$ in control homes. This includes a small number of staff in intervention and control homes who were vaccinated by their general practitioner or through a different occupational health service. Uptake was lower in part time staff: $21.2 \%$ and $18.4 \%$ in intervention homes and $4.0 \%$ and $4.0 \%$ in control homes. In both years the influenza A H3N2 subtype included in

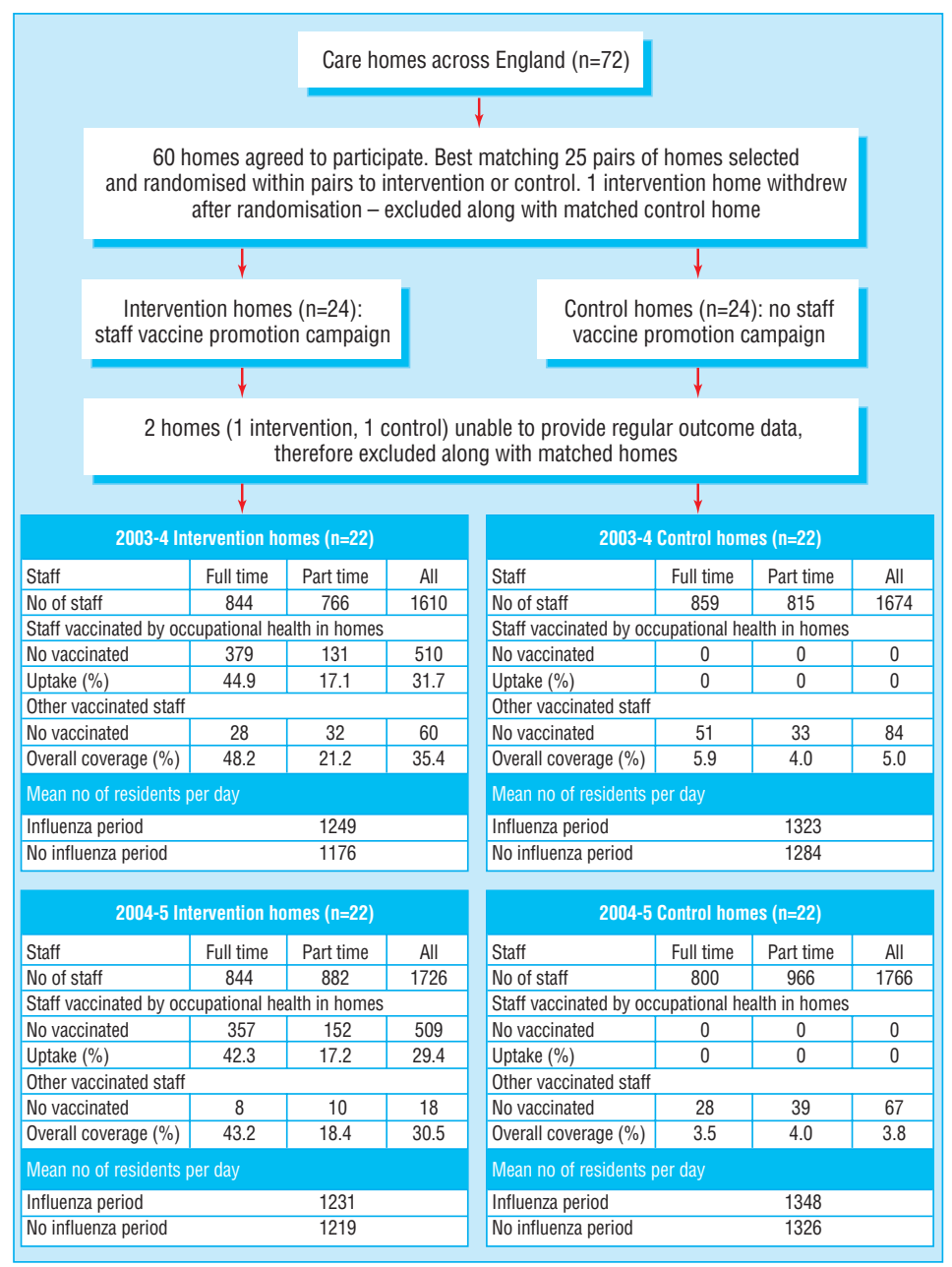

Fig 2 Participant flow 
Table 2 Numbers of outcome events and event rates in intervention and control homes

\begin{tabular}{|c|c|c|c|c|c|c|}
\hline & \multicolumn{2}{|c|}{ Intervention homes } & \multicolumn{2}{|c|}{ Control homes } & \multirow{2}{*}{ Weighted rate difference $(95 \% \mathrm{Cl})$} & \multirow{2}{*}{$P$ value } \\
\hline & No of events & Events per resident & No of events & Events per resident & & \\
\hline \multicolumn{7}{|l|}{ Year 1} \\
\hline \multicolumn{7}{|l|}{ Period of influenza activity: } \\
\hline No of residents & \multicolumn{2}{|c|}{$\mathrm{n}=1249^{*}$} & \multicolumn{2}{|c|}{$\mathrm{n}=1323^{*}$} & - & - \\
\hline Death & 140 & 0.112 & 203 & 0.153 & $-0.05(-0.07$ to -0.02$)$ & 0.002 \\
\hline Influenza-like illness & 142 & 0.114 & 300 & 0.227 & $-0.09(-0.14$ to -0.03$)$ & 0.004 \\
\hline $\begin{array}{l}\text { General practitioner } \\
\text { consultations for influenza-like } \\
\text { illness }\end{array}$ & 125 & 0.100 & 247 & 0.187 & $-0.07(-0.12$ to -0.02$)$ & 0.002 \\
\hline Admissions to hospital & 105 & 0.084 & 144 & 0.109 & $-0.02(-0.05$ to 0.02$)$ & 0.35 \\
\hline $\begin{array}{l}\text { Admissions with influenza-like } \\
\text { illness }\end{array}$ & 4 & 0.003 & 23 & 0.017 & $-0.02(-0.03$ to 0.00$)$ & 0.009 \\
\hline Death with influenza-like illness & 13 & 0.010 & 19 & 0.014 & $-0.01(-0.02$ to 0.01$)$ & 0.24 \\
\hline \multicolumn{7}{|l|}{ Period of no activity: } \\
\hline No of residents & \multicolumn{2}{|c|}{$\mathrm{n}=1176^{*}$} & \multicolumn{2}{|c|}{$\mathrm{n}=1284^{*}$} & - & - \\
\hline Death & 97 & 0.082 & 94 & 0.073 & $0.00(-0.03$ to 0.03$)$ & 0.93 \\
\hline Influenza-like illness & 114 & 0.097 & 114 & 0.089 & $0.00(-0.05$ to 0.05$)$ & 0.93 \\
\hline $\begin{array}{l}\text { General practitioner } \\
\text { consultations for influenza-like } \\
\text { illness }\end{array}$ & 87 & 0.074 & 99 & 0.077 & $-0.01 \quad(-0.06$ to 0.041$)$ & 0.74 \\
\hline Admissions to hospital & 77 & 0.065 & 86 & 0.067 & $0.00(-0.04$ to 0.03$)$ & 0.80 \\
\hline $\begin{array}{l}\text { Admissions with influenza-like } \\
\text { illness }\end{array}$ & 3 & 0.003 & 8 & 0.006 & $-0.01 \quad(-0.02$ to 0.01$)$ & 0.32 \\
\hline Death with influenza-like illness & 6 & 0.005 & 7 & 0.005 & $-0.01 \quad(-0.04$ to 0.02$)$ & 0.59 \\
\hline \multicolumn{7}{|l|}{ Year 2} \\
\hline \multicolumn{7}{|l|}{ Period of influenza activity: } \\
\hline No of residents & \multicolumn{2}{|c|}{$\mathrm{n}=1231^{*}$} & \multicolumn{2}{|c|}{$\mathrm{n}=1348^{*}$} & - & - \\
\hline Death & 99 & 0.080 & 123 & 0.091 & $-0.01 \quad(-0.04$ to 0.02$)$ & 0.49 \\
\hline Influenza-like illness & 149 & 0.121 & 179 & 0.133 & $0.00(-0.06$ to 0.06$)$ & 0.93 \\
\hline $\begin{array}{l}\text { General practitioner } \\
\text { consultations for influenza-like } \\
\text { illness }\end{array}$ & 124 & 0.101 & 155 & 0.115 & $-0.01 \quad(-0.07$ to 0.05$)$ & 0.77 \\
\hline Admissions to hospital & 93 & 0.076 & 102 & 0.076 & $-0.00(-0.03$ to 0.04$)$ & 0.84 \\
\hline $\begin{array}{l}\text { Admissions with influenza-like } \\
\text { illness }\end{array}$ & 12 & 0.010 & 9 & 0.007 & $0.00(-0.02$ to 0.02$)$ & 0.99 \\
\hline Death with influenza-like illness & 4 & 0.003 & 14 & 0.010 & $-0.01(-0.03$ to 0.00$)$ & 0.08 \\
\hline \multicolumn{7}{|l|}{ Period of no activity: } \\
\hline No of residents & \multicolumn{2}{|c|}{$\mathrm{n}=1219^{*}$} & \multicolumn{2}{|c|}{$\mathrm{n}=1326^{*}$} & - & - \\
\hline Death & 165 & 0.135 & 159 & 0.120 & $0.01(-0.03$ to 0.04$)$ & 0.70 \\
\hline Influenza-like illness & 247 & 0.203 & 243 & 0.183 & $0.03(-0.07$ to 0.12$)$ & 0.57 \\
\hline $\begin{array}{l}\text { General practitioner } \\
\text { consultations for influenza-like } \\
\text { illness }\end{array}$ & 177 & 0.145 & 211 & 0.159 & 0.00 (-0.08 to 0.08$)$ & 0.95 \\
\hline Admissions to hospital & 123 & 0.101 & 134 & 0.101 & $0.00(-0.03$ to 0.03$)$ & 0.86 \\
\hline $\begin{array}{l}\text { Admissions with influenza-like } \\
\text { illness }\end{array}$ & 12 & 0.010 & 8 & 0.006 & 0.01 (0.00 to 0.02$)$ & 0.31 \\
\hline Death with influenza-like illness & 12 & 0.010 & 6 & 0.005 & 0.01 (-0.01 to 0.02$)$ & 0.35 \\
\hline
\end{tabular}

*Average number of residents per day in homes during period.

the vaccine (Panama in year 1 and Fujian in year 2) differed from the circulating subtype, but antigenic similarities were sufficient for the vaccine to be protective.

Table 1 shows the baseline characteristics of residents in the homes and the median difference between matched pairs (intervention minus control homes). Intervention and control homes had similar baseline characteristics (table 1).

As the interaction between study year and intervention was significant results are reported separately for each year. Table 2 shows the number of outcome events during periods of influenza activity and no activity in intervention and control homes in 2003-4 and 2004-5. The average number of residents in intervention and control homes and event rates per resident period are shown for each period. Table 2 also includes the results of the meta-analysis.

Intervention homes had significantly lower all cause mortality during the influenza period of 2003-4 (rate difference per 100 residents per period $-5.0,95 \%$ confidence interval -7.0 to
$-2.0 ; \mathrm{P}=0.002)$ compared with control homes (number needed to treat $8.2,5.8$ to 20.4). The effect was not seen during periods of no influenza activity or in the 2004-5 influenza period when influenza levels were low. In the influenza period of 2003-4 significantly lower rates were found for the secondary outcomes in intervention homes compared with control homes: influenzalike illness (rate difference per 100 residents per period -9.0, $95 \%$ confidence interval -14.0 to $-3.0 ; \mathrm{P}=0.004$, number needed to treat $4.5,2.9$ to 13.6), general practitioner consultations for influenza-like illness $(-7.0,-12.0$ to -2.0 ; $\mathrm{P}=0.002$, number needed to treat $5.8,3.4$ to 20.4), and admissions to hospital with influenza-like illness $(-2.0,-3.0$ to $0 ; \mathrm{P}=0.009$, number needed to treat $20.4,13.6$ to 102.1 ). Evidence was found of significant heterogeneity of results for influenza-like illness and associated general practitioner consultations (see bmj.com). No significant differences were found in secondary outcome measures during any other period. The corresponding forest plots are on bmj.com. 


\section{Discussion}

Vaccinating care home staff against influenza can prevent deaths in residents, morbidity, and associated health service use during periods of moderate influenza activity. The reduction is equivalent to preventing five deaths, two admissions to hospital with influenza-like illness, seven general practitioner consultations for influenza-like illness, and nine cases of influenza-like illness per 100 residents during the period of influenza activity. The numbers of staff vaccinations needed to prevent one death, one case of influenza-like illness, one general practitioner consultation for influenza-like illness, and one admission to hospital with influenza-like illness were $8,5,6$, and 20. These effects were seen despite high levels of vaccination of residents (poor immune response to vaccine in elderly people can often leave them vulnerable to influenza). In addition to the reductions in mortality and morbidity, the intervention has the potential to substantially reduce health service costs in years with moderate levels of influenza activity, and especially during epidemics.

Lead nurses were not blinded to the intervention, as introducing a placebo arm would have diminished participation rates and would not have been compatible with running a vaccine promotion campaign. We deliberately chose a primary outcome measure (all cause mortality) that is not subject to observer bias, and powered the study accordingly. Nurses in intervention homes might have been less likely to label residents' illnesses as influenza if they strongly believed that the intervention protected residents. This would have led to lower rates of influenza-like illness in intervention homes throughout the study period, not just during the period of influenza activity. Conversely nurses in intervention homes might have been more likely to detect influenza because the vaccination campaign would have raised their awareness.

The intervention was randomly assigned and baseline characteristics of residents in intervention and control homes showed no significant differences that could have accounted for the observed effect. The $4 \%$ higher uptake of vaccination in residents in intervention homes could not have accounted for the $25 \%$ decrease in mortality or a halving of the influenza-like illness rate. The observed heterogeneity of effect size for influenza-like illness and associated general practitioner consultations is to be expected as the effect depends on introductions of influenza that are stochastic events. Because of heterogeneity we used a random effects model to produce the summary effect estimates.

Influenza activity in 2004-5 was among the lowest recorded since $1988 .^{23}$ Nearly twice as much influenza-like illness was reported in 2003-4 as in 2004-5. Because the effect size should be related to the level of circulating influenza we made an a priori decision to analyse the effect separately in the two years. This was supported by a significant interaction between year and intervention on mortality and other outcomes. The direction of effect is the same in both years but the effect is much greater in the first year when influenza activity was substantially higher. The lack of a statistically significant effect in a year with exceptionally low influenza activity is consistent with the hypothesis that the vaccination of staff prevents influenza related morbidity and mortality in residents. Indeed if we had found similar effect sizes in two years with noticeably different levels of influenza activity this would not have been consistent with the hypothesis. The fact that an effect was shown in a year with below average influenza activity suggests that a protective effect would be observed most years. Theoretically the benefits would be substantially greater in epidemic years. The effect might also have been greater if the circulating influenza strain had matched the vaccine strain more closely. Achieving higher vaccine uptake could also have increased effectiveness but is notoriously difficult in healthcare workers. In England's national health service trusts uptake is typically around $15 \% .{ }^{25}$ Our uptake in full time staff was $48.2 \%$ (2003-4) and 43.2\% (2004-5) Theoretically better vaccine uptake could have prevented an even greater burden of disease.

A recent systematic review ${ }^{26}$ of influenza vaccination of healthcare workers to reduce influenza related outcomes in high risk patients identified only two relevant studies; the first was a pilot for the second. ${ }^{17}{ }^{18}$ The main study showed a reduction in mortality from $22.4 \%$ to $13.6 \%$ over a six month period, with unusually high influenza activity (Royal College of General Practitioners' influenza-like illness rates peaked at 220 per 100000 ). The average vaccine uptake in patients was $48 \%$ in intervention wards and 33\% in control wards and uptake of vaccine by staff was $51 \%$. After controlling for differences in baseline characteristics the odds ratio for mortality was 0.61 (95\% confidence interval 0.36 to $1.04 ; \mathrm{P}=0.09$ ). The mortality in our study over the three months in which influenza was circulating in 2003-4 was 15 per 100 residents in control homes and 11 per 100 residents in intervention homes. Our study also showed an important effect on mortality, but this was apparent despite much lower levels of influenza and higher vaccine uptake by residents. No other studies were identified with patient mortality as the primary outcome. One observational study found significantly lower influenza-like illness rates in homes with higher uptake of vaccine by staff even after controlling for vaccine uptake by residents. ${ }^{27}$ Another study linked rising rates of hospital staff vaccination to falling rates of nosocomial influenza but could not rule out other causes for the decline. ${ }^{28}$

This study provides strong evidence to support influenza vaccination of care home staff even when vaccine uptake by residents is high. Results are likely to be generalisable to other care homes in the United Kingdom and abroad and may also be applicable to acute hospital settings, in particular elderly care and rehabilitation wards. It has proved difficult to achieve high uptake rates in healthcare workers owing to perceptions that influenza is a relatively trivial illness, concern about side effects, beliefs that the vaccine is ineffective, and lack of time and motivation..$^{29}$ Campaigns to promote influenza vaccination among healthcare workers or staff of long term care facilities should emphasise the protection of vulnerable patients and residents as well as the benefits to the individual.

We thank the lead nurses in the care homes who helped to implement the intervention and collect the data.

Contributors: All authors were substantially involved in the conception and design of the study, or analysis and interpretation of data, drafting the article or revising it critically for important intellectual content, and final approval of the version to be published. ACH was the principal investigator and is guarantor

Funding: Department of Health, which had no role in the collection, analysis, or interpretation of data; the writing of the report; or the decision to submit the paper for publication. ACH is funded by Camden primary care trust.

Competing interests: None declared.

Ethical approval: This study was approved by the London multicentre research ethics committee (No 02/2/56).

1 McBean AM, Hebert PL. New estimates of influenza-related pneumonia and influenza hospitalizations among the elderly. Int J Infect Dis 2004;8:227-35.

Bradley SF. Prevention of influenza in long-term-care facilities. Infect Control Hosp Epidemiol 1999;20:629-37.

3 Morens DM, Rash VM. Lessons from a nursing home outbreak of influenza A. Infect Control Hosp Epidemiol 1995;16:275-80.

4 Staynor K, Foster G, McArthur M, McGeer A, Petric M, Simor AE. Influenza A outbreak Staynor K, Foster G, McArthur M, McGeer A, Petric M, Simor AE. Influenza A outbreak
in a nursing home: the value of early diagnosis and the use of amantadine hydrochloride. Can J Infect Control 1994;9:109-11. 


\section{Research}

5 Coles B, Balzano GJ, Morse DL. An outbreak of influenza A (H3N2) in a well immunised nursing home population. J Am Geriatr Soc 1992;40:589-92.

6 Patriarca PA, Weber JA, Parker RA, Hall WN, Kendal AP, Bregman DJ, et al. Efficacy of influenza vaccine in nursing homes. Reduction in illness and complications during an influenza A (H3N2) epidemic. JAMA 1985;253:1136-9.

7 Anon. An influenza A outbreak in an Ontario nursing home: estimates of vaccine efficacy. Can Comm Dis Report 1995;21:61-4.

8 Centers for Disease Control and Prevention. Impact of influenza on a nursing home population-New York. MMWR 1983;32:32-4.

9 Jefferson T, Rivetti D, Rivetti A, Rudin M, Di Pietrantonja C, Demicheli V. Efficacy and effectiveness of influenza vaccines in elderly people: a systematic review. Lancet 2005;366:1165-74.

10 Ohmit SE, Arden NH, Monto AS. Effectiveness of inactivated influenza vaccine among nursing home residents during an influenzas type A(H3N2) epidemic. J Am Geriatr Soc 1999;47:165-71.

11 Monto AS, Hornbuckle K, Ohmit SE. Influenza vaccine effectiveness among elderly nursing home residents: a cohort study. Am J Epidemiol 2001;154:155-60.

12 Beyer WE, Palache AM, Baljet M, Masurel N. Antibody induction by influenza vaccination in the elderly: a review of the literature. Vaccine 1989;7:385-94.

13 Remarque EJ, Cools HJM, Boere TJ, van der Kils RJ, Masurel N, Ligthart GL Functional disability and antibody response to influenza vaccine in elderly patients in Dutch nursing home. BMJ 1996;312:1015-8.

14 Van Essen GA, Palache AM, Forleo E, Fedson DS. Influenza vaccination in 2000 recommendations and vaccine use in 50 developed and rapidly developing countries Vaccine $2003 ; 21: 1780-5$

15 Department of Health. The influenza immunisation programme. PL/CMO/2005/9 www.dh.gov.uk/PublicationsAndStatistics/LettersAndCirculars/ProfessionalLetters/ ChiefMedicalOfficerLetters/ChiefMedicalOfficerLettersArticle/fs en?CONTENT_ID = 4116516\&chk = qsKsaR (accessed 31 Oct 2006).

\section{What is already known on this topic}

Vaccinating elderly people against influenza reduces sickness and death rates but provides incomplete protection because the immunological response to vaccine is often suboptimal

Two randomised controlled trials of limited size on elderly care wards with low vaccine coverage suggest that vaccinating staff against influenza can reduce death rates during periods of high influenza activity

\section{What this study adds}

Vaccinating care home staff against influenza can prevent deaths in residents, morbidity, and associated health service use during periods of moderate influenza activity

The intervention is effective even when there are high levels of vaccination of residents and incomplete vaccine coverage in staff
16 Wilde JA, McMillan JA, Serwint J, Butta J, O'Riordan MA, Steinhoff MC. Effectivenes of influenza vaccine in health care professionals: a randomized trial. JAMA 1999-281:908-13.

17 Potter J, Stott DJ, Roberts MA, Elder AG, O’Donnell BO, Knight PV, et al. Influenza vaccination of health care workers in long-term-care hospitals reduces the mortality of elderly patients. J Infect Dis 1997;175:1-6.

18 Carman WF, Elder AG, Wallace LA, McAulay K, Walker A, Murray GD, et al. Effects of influenza vaccination of health-care workers on mortality of elderly people in long-term care: a randomised controlled trial. Lancet 2000;355:93-7.

19 Nicholson KG. Should staff in long-stay hospitals for elderly patients be vaccinated against influenza? Lancet 2000;355:83-4.

20 Donner A, Klar N. Design and analysis of cluster randomization trials in health research London: Arnold, 2000.

21 Campbell MK, Elbourne DR, Altman DG. CONSORT statement: extension to cluster randomised trials BMJ 2004;328:702-8.

22 Kerry SM, Bland JM. Trials which randomize practices II: sample size? Fam Prac 1998;15:84-7.

23 Health Protection Agency. Epidemiological data-influenza. wwwhpa.org.uk/ infections/topics_az/influenza/seasonal/flureports0405.htm (accessed 31 Oct 2006).

24 Thompson SG, Pyke SDM, Hardie RJ. The design and analysis of paired cluster randomized trials: an application of meta-analysis techniques. Stat Med 1997;16:2063-79.

25 Elgohari S, Joseph CA, Goddard NL. Three years' experience of monitoring influenza vaccine uptake in healthcare workers in acute hospital trusts in Fngland 2001/02-2003-04. Five Nations Health Protection Conference, 2004.

26 Burls A, Jordan R, Barton P, Olowokure B, Wake B, Albon E, et al Vaccinating healthcare workers against influenza to protect the vulnerable-is it a good use of healthcare resources? A systematic review of the evidence and an economic evaluation. Vaccine 2006;8:4212-21.

27 Saito R, Suzuki H, Oshitani H. The effectiveness of influenza vaccine against influenza A (H£N”) virus infections in nursing homes in Nigata, Japan, during the 1998-1999 and 1999-2000 seasons. Inf Control Hosp Epidemiol 2002;23:82-4.

28 Salgado CD, Giannetta ET, Hayden FG, Farr BM. Preventing nosocomial influenza by improving the vaccine acceptance rate of clinicians. Infect Control Hosp Epidemiol 2004;25:923-8.

29 Steiner M, Vermeulen LC, Mullahy J, Hatney M. factors influencing decisions regarding influenza vaccination and treatment: a survey of healthcare workers. Infect Control Hos Epidemiol 2002;23:625-7.

(Accepted 10 October 2006)

doi $10.1136 /$ bmj. 39010.581354 .55

University College London Centre for Infectious Disease Epidemiology, Department of Primary Care and Population Sciences, London NW3 2PF

Andrew C Hayward senior lecturer in infectious disease epidemiology

Richard Harling honorary lecturer

Sally Wetten research assistant

Anne M Johnson professor of infectious disease epidemiology

Shahed Murad statistician

Westminster Healthcare, Leatherhead, Surrey

Susan Munro senior nurse manager

Medical Research Council Epidemiology Resource Centre, Southampton General Hospital

Julia Smedley homorary senior lecturer

Health Protection Agency Centre for Infections, London

John M Watson consultant epidemiologist

Correspondence to: A Hayward a.hayward@pcps.ucl.ac.uk 